\title{
Aplicação do método por digitalização 3D para obtenção de medidas antropométricas no Modelo Humano Digital
}

\author{
Application of the 3D scanning method to obtain anthropometric measurements in \\ the Digital Human Model
}

BRENDLER, Clariana; Doutorado; Universidade Federal do Rio Grande do Sul clariana.brendler@ufrgs.br

TEIXEIRA, Fábio; Pós-Doutorado; Universidade Federal do Rio Grande do Sul fabiogt@ufrgs.br

ZUBARAN, Gabriela; Doutorado; Universidade Federal do Rio Grande do Sul gabrielapizzato@gmail.com

MELLO, Guilherme; Graduando; Universidade Federal do Rio Grande do Sul guilherme.mello@ufrgs.br

\section{Resumo}

O objetivo deste trabalho é apresentar uma aplicação do método de obtenção de parâmetros antropométricos estáticos por meio de um digitalizador 3D de baixo custo (BRENDLER, 2013) e descrever as implicações e contribuições ocorridas neste processo. Os parâmetros antropométricos são obtidos e inseridos num modelo humano digital (MHD) para comparação e análise dos resultados em relação às medidas do modelo 3D obtidas pela digitalização. Foram utilizados dois participantes, um do sexo feminino e um do masculino, representando o percentil estatura $5 \%$ e $95 \%$ respectivamente. Os modelos 3D dos participantes foram adquiridos com uso do dispositivo Kinect da Microsoft. Os resultados obtidos foram extremamente compatíveis o que demonstra a viabilização do método utilizando a digitalização para obtenção de medidas antropométricas para aplicação no MHD. Otimizando o processo de medição, reduzindo o tempo necessário de mensuração com o usuário e aumentando a precisão das medidas antropométricas para auxiliar nas análises ergonômicas do produto.

Palavras Chave: Digitalização 3D de baixo custo; Antropometria; Análise Ergonômica; MHD.

\begin{abstract}
The objective of this work is to present an application of the method of obtaining static anthropometric parameters by means of a low-cost 3D scanner (BRENDLER, 2013) and to describe the implications and contributions of this process. The anthropometric parameters are obtained and inserted in a digital human model (MHD) for comparison and analysis of the results in relation to the measurements of the 3D model obtained by the digitalization. Two participants, one female and one male, were used, representing the percentile height 5\% and 95\% respectively. The participants' 3D models were purchased using the Microsoft Kinect device. The results obtained were extremely compatible which demonstrates the viability of the method using the digitization to obtain anthropometric measurements for application in the MHD. Optimizing the measurement
\end{abstract}


process, reducing the time needed to measure with the user and increasing the accuracy of the anthropometric measurements to aid in the ergonomic analysis of the product.

Keywords: Low-cost 3D scanning; Anthropometry; Ergonomic Analysis; MHD.

\section{Introdução}

As análises ergonômicas são realizadas durante as etapas de desenvolvimento de projetos de produtos para avaliar questões como conforto, segurança e eficácia. Estas análises são praticadas cada vez mais em ambientes virtuais por questões de otimização do processo, melhorias no resultado final e diminuição dos custos de projeto. Assim, o uso de modelos humanos digitais (MHD) é fundamental, pois permitem estas análises em ambiente virtual, eliminando a participação de usuários reais e de protótipos físicos em escala real.

Os parâmetros antropométricos são inseridos no MHD para que estes representem as características físicas dos usuários durante o uso do produto. As questões versam sobre a necessidade de parâmetros antropométricos para produtos personalizados ou produtos utilizados por uma gama de usuários que representam uma determinada população bem como estes parâmetros são obtidos. Dependendo da necessidade de projeto, há duas maneiras para obtenção destes parâmetros antropométricos: por método direto (medições manuais) ou método indireto (medições através de digitalizadores tridimensionais).

Até o início dos anos 2000, a obtenção dos parâmetros antropométricos limitava-se à medição manual utilizando instrumentos tradicionais como balança de precisão, paquímetro e fitas métricas. Porém, os métodos e procedimentos de obtenção de medidas do corpo humano por métodos manuais são considerados procedimentos demorados e envolvem o contato físico com os indivíduos a serem mensurados (LU e WANG, 2008; SIMMONS, 2001; TOMKINSON e SHAW, 2013). O contato físico do antropometrista com o indivíduo a ser mensurado e o tempo demasiado para a realização do levantamento dos parâmetros antropométricos são um problema, principalmente, em indivíduos com deficiência física, idosos e grávidas, devido às dificuldades em se manter na posição estática por um tempo prolongado. Em pessoas com deficiências, que possuem deformação física, as medições tornam-se complexas e, em algumas variáveis, os parâmetros antropométricos são impossíveis de serem obtidos. O tipo de deficiência física pode afetar consideravelmente as distribuições das dimensões do corpo (HOBSON e MOLENBROEK, 1990; JONES e RIOUX, 1997; LUXIMON et al., 2012).

Em muitos casos, conforme a deficiência física do indivíduo a ser mensurado, o levantamento antropométrico é realizado através da obtenção de um molde de gesso, para que sejam obtidas as medidas antropométricas no próprio molde. Este procedimento pode causar um constrangimento aos envolvidos e, dependendo do clima, esse tipo de situação é agravado por causa do frio e do gesso molhado em contato com a pele do indivíduo, o que causa grande desconforto (BRENDLER, 2013). Com o avanço da tecnologia, pesquisas vêm sendo realizadas para a obtenção de parâmetros antropométricos por meio de digitalizadores 3D, para que seja possível a aquisição dos parâmetros antropométricos sem o contato físico com o indivíduo a ser mensurado, conforme a identificação da necessidade de medidas mais rápidas, precisas e sem o contato físico no usuário (D'APUZZO, 2009; TONG et al., 2012).

Brendler (2013) desenvolveu um método em que obtém parâmetros antropométricos 
estáticos utilizando um digitador 3D de baixo custo, o Kinect da Microsoft. Este método possibilita a geração do modelo 3D do usuário e, dessa forma, os parâmetros antropométricos são obtidos diretamente no modelo 3D gerado. Assim como há a inserção da tecnologia para aprimorar o processo de obtenção dos parâmetros antropométricos, também pode ser utilizada para auxiliar nas etapas de análises ergonômicas de projeto de produto. Por exemplo, as análises ergonômicas realizadas em ambiente virtual, nas quais utilizam um MHD, seriam facilitadas se o método de Brendler (2013) fosse utilizado para otimizar e possibilitar a inserção dos parâmetros antropométricos no MHD. Portanto, o objetivo deste trabalho é apresentar uma aplicação do método de Brendler (2013), em que os parâmetros antropométricos obtidos são inseridos no MHD. Assim, descrever as implicações e contribuições ocorridas neste processo. Os parâmetros antropométricos são inseridos no MHD para realização de análises ergonômicas em ambiente virtual o que favorece o desenvolvimento de produtos que contemplam os requisitos ergonômicos de projeto como o conforto, a segurança e eficácia.

\subsection{Método de Brendler (2013)}

O método de Brendler (2013) é dividido em duas etapas: a obtenção do modelo 3D do usuário e o levantamento dos parâmetros antropométricos a partir do modelo 3D gerado (Figura 01). Para o processo de geração do modelo 3D, o indivíduo permanece parado durante a varredura das imagens. Após a digitalização, é iniciado o processo de montagem das malhas tridimensionais. A Figura 2 apresenta o processo de alinhamento das malhas tridimensionais e, em amarelo, o corte das imagens do piso. Para o processo da digitalização 3D, o operador do Microsoft Kinect se movimenta em torno do indivíduo que permanece parado. Desta forma, é realizada a varredura das imagens em diferentes ângulos, completando $360^{\circ}$ em torno do indivíduo.

Figura 1 - Geração do modelo 3D e finalização do modelo 3D.

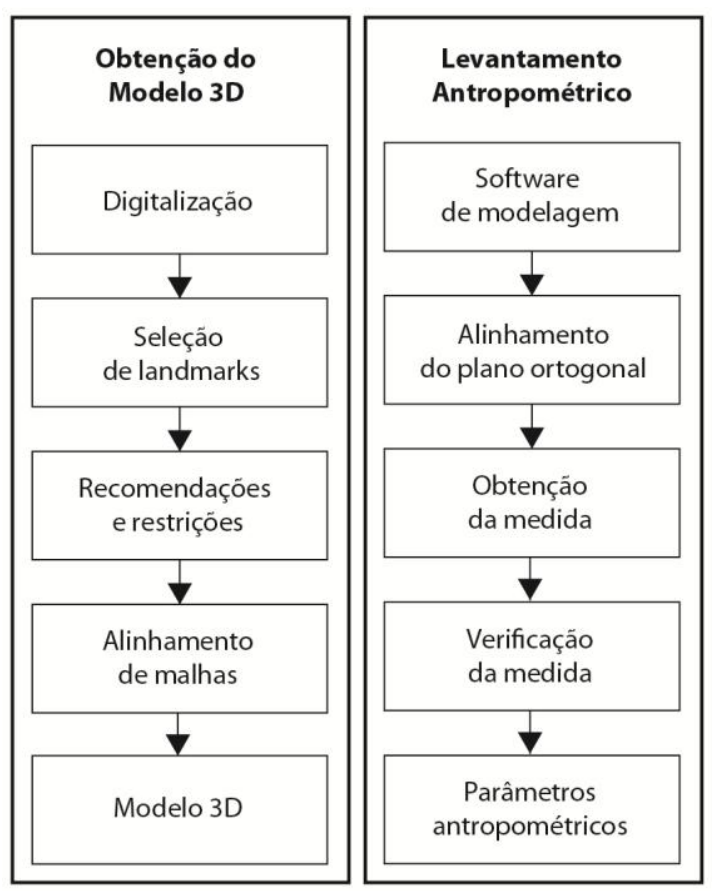

Fonte: Brendler (2013) 
Figura 2 - Geração do modelo 3D e finalização do modelo 3D.

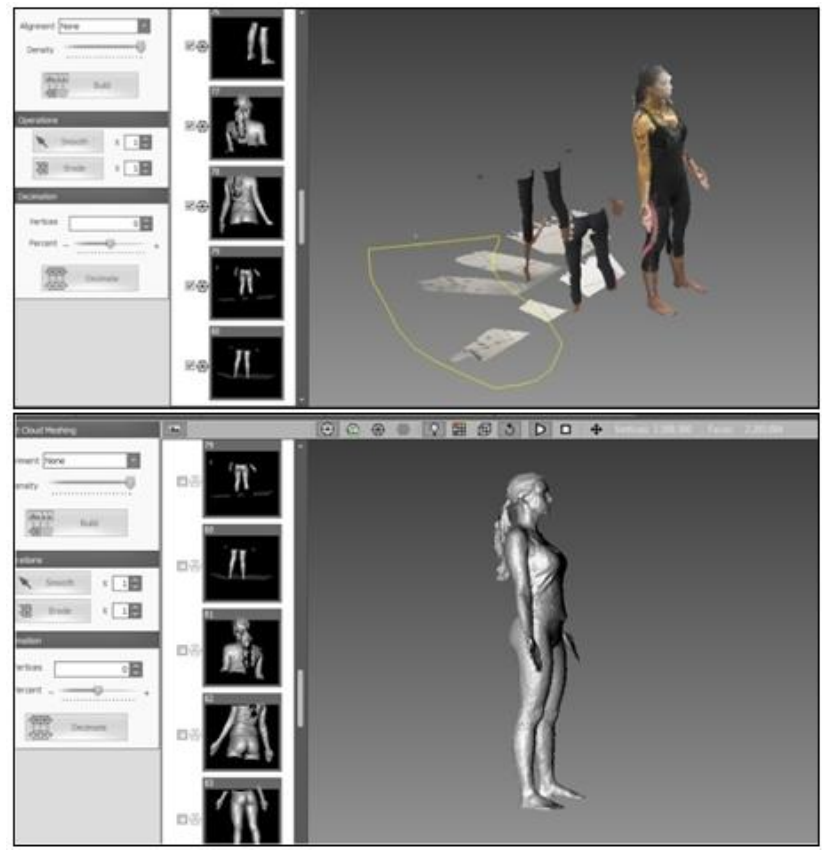

Fonte: Brendler (2013)

A obtenção dos parâmetros antropométricos é realizada diretamente no modelo 3D. O indivíduo deve se manter parado durante a digitalização das imagens pelo Kinect. Se o indivíduo alterar a posição do braço ou da cabeça, por exemplo, no momento da varredura, é gerado um modelo 3D com imperfeições na sua configuração. A tolerância, portanto, para a movimentação do indivíduo durante a digitalização se restringe à respiração. A Figura 3 apresenta imagens do levantamento antropométrico realizado a partir do modelo 3D obtido. Realiza-se o alinhamento do modelo 3D aos eixos $x$, y e z. A partir do alinhamento, é criado um Box. Desta forma, inicia-se o processo de construção de linhas de referência para a obtenção dos parâmetros antropométricos, conforme é apresentado na Figura 03.

Figura 3 - levantamento antropométrico a partir do modelo 3D.

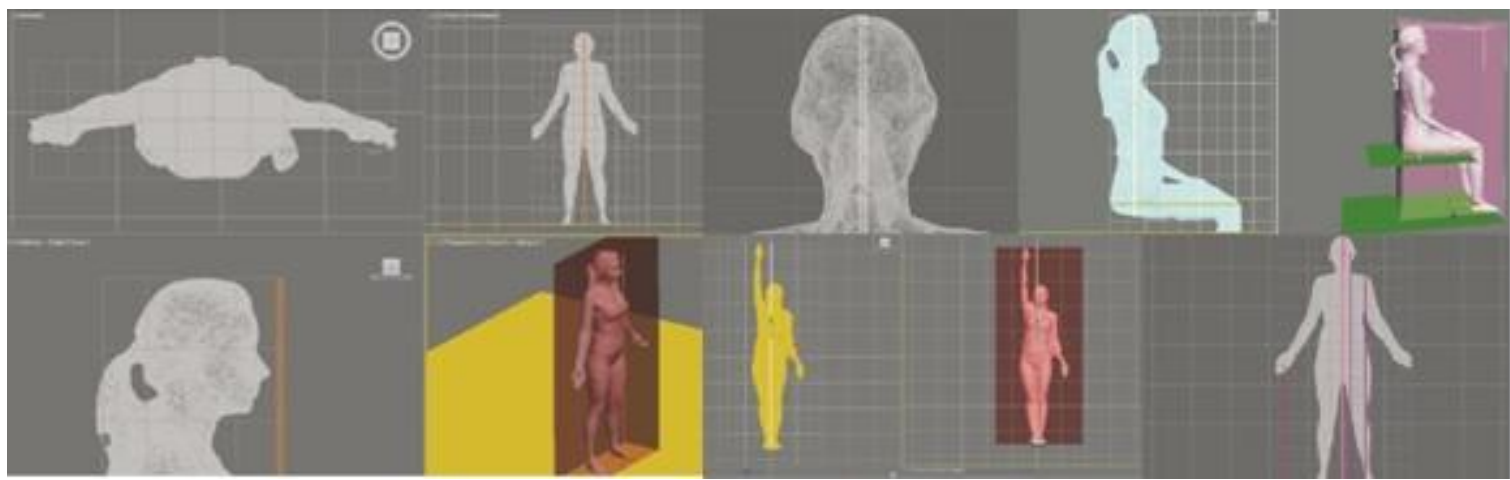

Fonte: Brendler e Teixeira (2016) 


\subsection{Análise ergonômica virtual com uso do Modelo Humano Digital}

Para que um determinado produto seja ergonômico, este deve contemplar os principais requisitos que compreendem a ergonomia física como o conforto, a segurança e a eficácia. Para isso, no processo de PDP estes requisitos são hierarquizados ainda nas etapas iniciais de projeto. Para que estes produtos contemplem os requisitos ergonômicos, são realizadas análises ergonômicas por meio de análises do uso dos produtos, que têm como objetivo auxiliar na verificação do dimensionamento correto do produto por meio dos parâmetros antropométricos estáticos e dinâmicos dos usuários (IIDA, 2005; KOO et al., 2015). As análises ergonômicas em meio virtual com uso de MHD facilitam o processo de análise, possibilitando a avaliação tanto nas etapas iniciais de projeto quanto nas etapas finais de detalhamento e definição da solução final do produto. $\mathrm{O}$ uso do MHD no PDP pode eliminar a produção de protótipos físicos durante as etapas do projeto, minimizando os custos, o tempo e os possíveis erros que podem ocorrer no dimensionamento do produto (SANTOS et al., 2009; IIDA, 2005 e BLANCHONETTE, 2010).

Por exemplo, nas análises ergonômicas virtuais o produto a ser desenvolvido é modelado em 3D e, na etapa de projeto informacional, são definidas as informações necessárias para o desenvolvimento do produto e o público-alvo (os usuários que irão utilizar estes produtos). A definição dos usuários é de extrema importância para determinar os parâmetros antropométricos estáticos aplicados no MHD para as realizações das tarefas, assim como, os requisitos de projeto segundo as necessidades dos usuários. Para que estes produtos atendam aos requisitos ergonômicos de projeto, o usuário deve executar as tarefas com os ângulos de conforto nas articulações do corpo dentro do recomendado pela base teórica. Para isso, o MHD, representando estes usuários antropometricamente, deve realizar as tarefas prescritas e as recomendações ergonômicas são elaboradas e aplicadas no PDP.

Figura 4 - levantamento antropométrico a partir do modelo 3D.

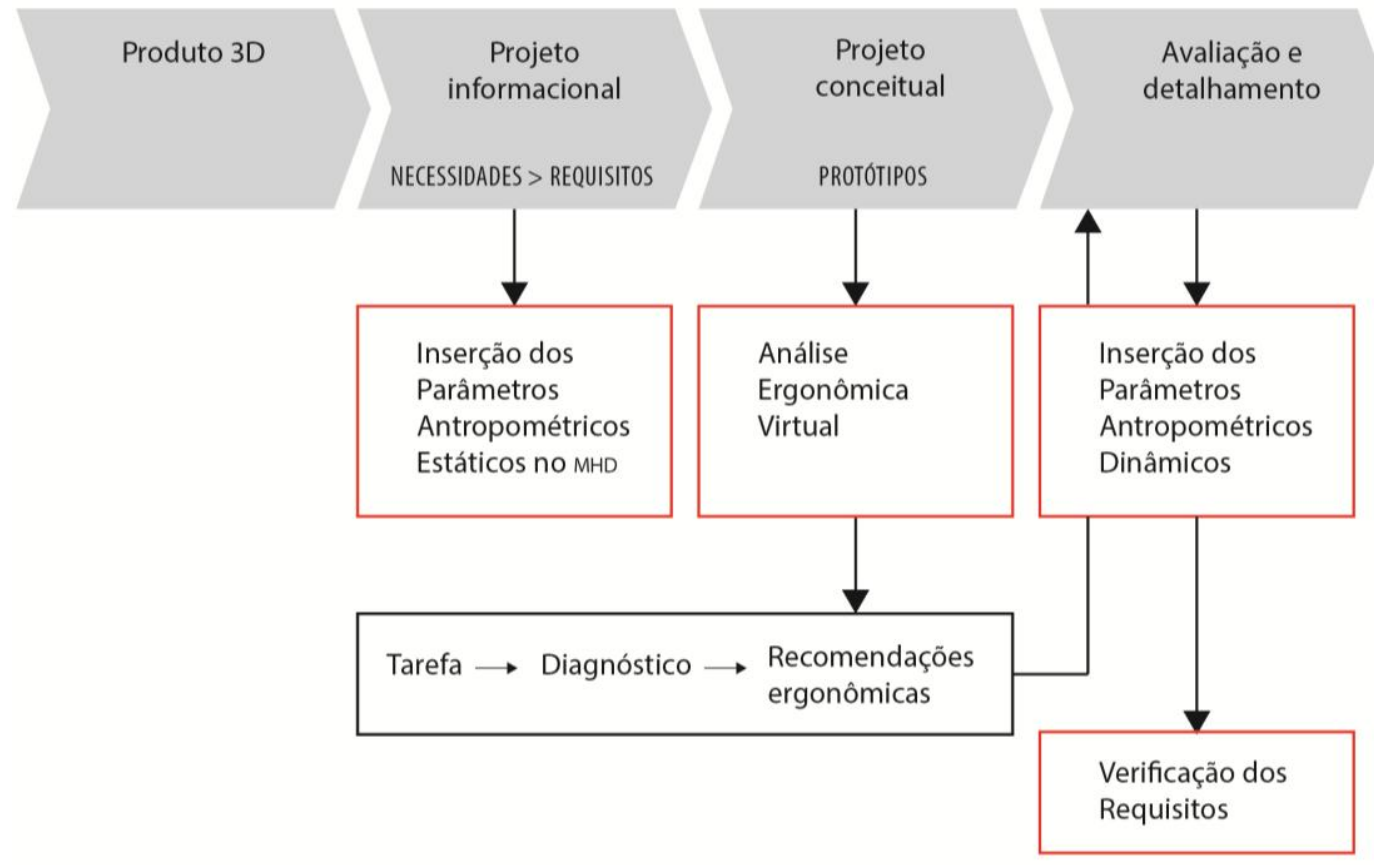

Fonte: Brendler (2017) 
Por exemplo, as recomendações ergonômicas para o desenvolvimento do produto para uma gama de usuários são elaboradas a partir das análises ergonômicas com o MHD representando a altura mínima e a máxima do usuário, para que todos os usuários dentro desta faixa de medidas possam utilizar estes produtos com conforto e segurança. Isso é possível a partir da análise da tarefa, em que são avaliadas a postura dos usuários, os ângulos de conforto e os alcances, verificados na etapa definida como diagnóstico e as recomendações ergonômicas são definidas e aplicadas na etapa de avaliação e detalhamento do desenvolvimento do produto. Sendo assim, este processo de análise ergonômica com uso do MHD é apresentado graficamente na Figura 4.

\section{Métodos e Processos}

Para a obtenção de medidas antropométricas estáticas por meio de um digitalizador 3D de baixo custo e inserção destas medidas no MHD foi utilizado o método Brendler (2013) e o MHD desenvolvido no trabalho de tese de Brendler (2017). O MHD foi desenvolvido para ser utilizado em análises ergonômicas em ambiente virtual e possibilitar não apenas a obtenção de medidas antropométricas estáticas como também as dinâmicas. Medidas estas fundamentais para que se desenvolvam produtos ergonômicos que contemplem os requisitos de projeto como o conforto, a segurança e a eficácia. A Figura 5 apresenta graficamente as etapas do processo desenvolvidas no presente artigo.

Figura 5 - levantamento antropométrico a partir do modelo 3D.

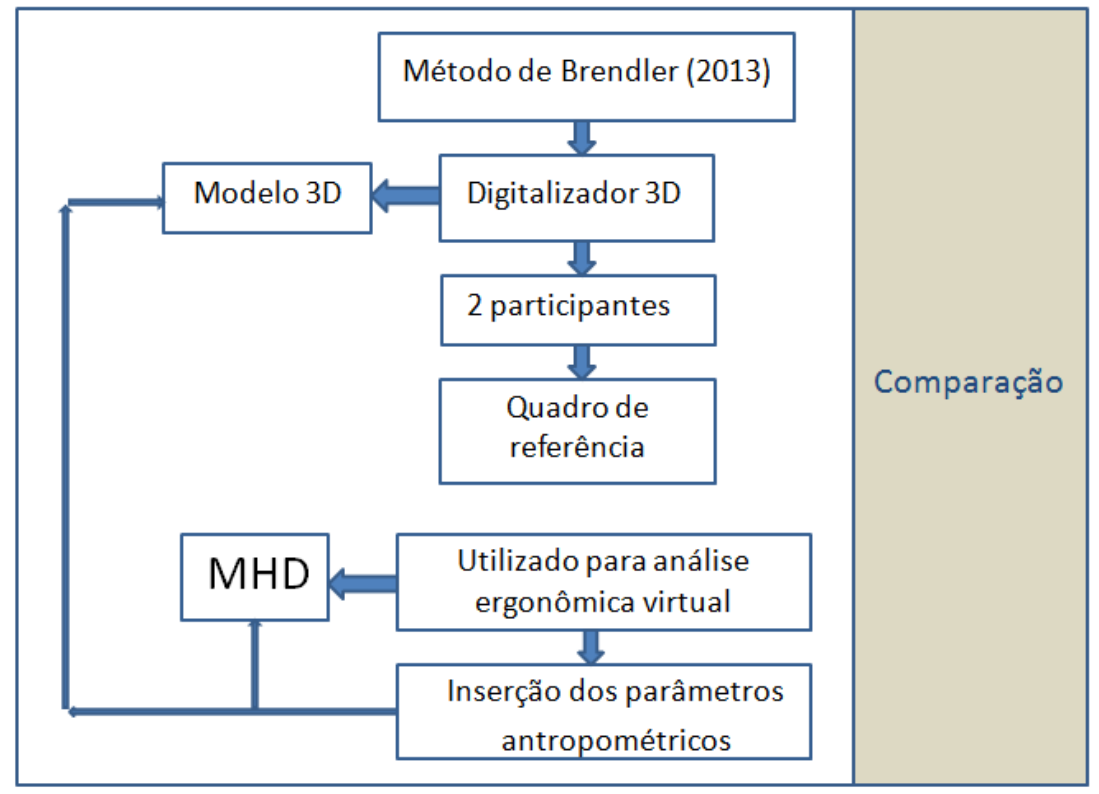

Fonte: Elaborado pelos autores.

Foram utilizados dois participantes, um do sexo feminino, representando o percentil estatura $5 \%$ e um do sexo masculino, representando o percentil estatura $95 \%$. Estes foram digitalizados e, dessa forma, o modelo 3D foi gerado. A partir do modelo 3D, as medidas antropométricas foram obtidas e após, inseridas no MHD paramétrico. Este processo de obtenção de medidas é descrito detalhadamente bem como a inserção destas medidas para uso em análises ergonômicas em ambiente virtual nos itens 2.1 e 2.2 do presente artigo. 


\subsection{Digitalização e obtenção das medidas antropométricas}

O método de obtenção das medidas antropométricas estáticas utiliza um sistema de digitalização 3D contendo um scanner 3D - Kinect da Microsoft e, para a obtenção de detalhes como as medidas das mãos, dos pés e do rosto, é utilizado um scanner 3D de alta resolução, o Artec Eva. O processo de digitalização 3D tem uma duração de aproximadamente 1 minuto para o corpo inteiro em cada participante e, aproximadamente, 30 segundos para cada segmento como as mãos, os pés e o rosto. Os participantes permanecem imóveis e na posição anatômica enquanto é realizada a captura das imagens. Durante a digitalização 3D dos participantes da pesquisa, o software gera o modelo 3D em tempo real. Após, é necessário realizar o processamento deste para o preenchimento de algumas falhas e ruídos na malha 3D. 0 processamento dos modelos 3D não necessita da participação dos usuários da pesquisa e tem uma duração de aproximadamente 5 horas de trabalho para os modelos 3D gerados para a presente pesquisa (o tempo para o processamento dos modelos 3D depende do nível de experiência de quem está processando e da qualidade do computador).

O scanner Kinect é um scanner 3D de baixo custo, e tem sido muito utilizado em pesquisas em todo o mundo nas áreas de ergonomia, saúde, tecnologia e design, animação e games, por ser de fácil uso, leve e portátil. Entretanto, para a resolução de geometrias complexas e pequenas, como os detalhes do rosto, mãos e pés é necessário utilizar um scanner 3D com alta resolução dimensional como os scanners 3D com sistemas de luz branca ou a laser. Para a presente pesquisa, foi utilizado o scanner 3D de luz branca para a geração do modelo 3D da mão, do pé e do rosto, tanto para o participante feminino como para o masculino. Para a geração do modelo 3D de corpo inteiro, foi utilizado o scanner por sistema de infravermelho (kinect). Desta forma, os modelos 3D das mãos, dos pés e do rosto, são inseridos no modelo 3D de corpo inteiro. Para isso, foi utilizado o software Geomagic Qualify , o qual possibilita o processo de alinhamento entre os pontos das malhas 3D e da compatibilidade entre ambas.

No momento da varredura da imagem, o primeiro participante representando o percentil $5 \%$ de altura feminino, prendeu o cabelo e a vestimenta utilizada pelo participante foi uma calça e uma blusa justa ao corpo. A Figura 6 apresenta o modelo 3D da mão direita e o o modelo 3D do pé direito referente ao participante feminino. 0 modelo 3D do corpo inteiro com o rosto, os pés e as mãos inseridos é apresentado na Figura 7.

Figura 6 - Modelo 3D da mão e do pé direito.

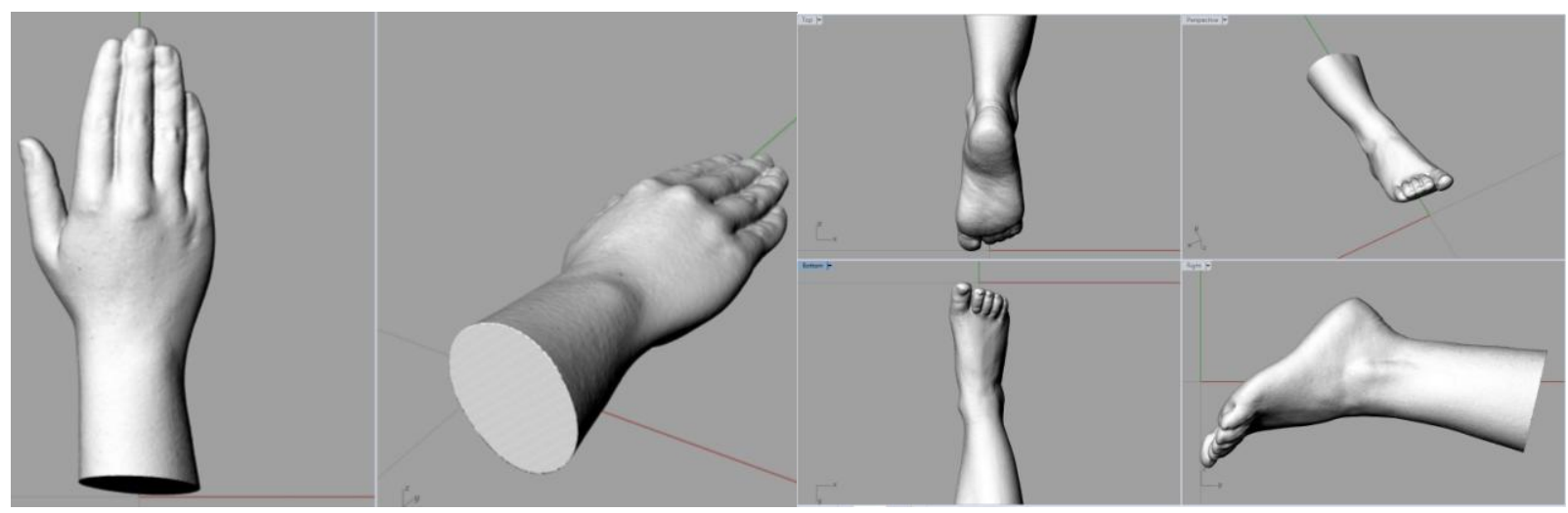

Fonte: Elaborado pelos autores. 
Figura 7 - Modelo 3D do corpo inteiro da participante representando o percentil 5\% de altura feminino.

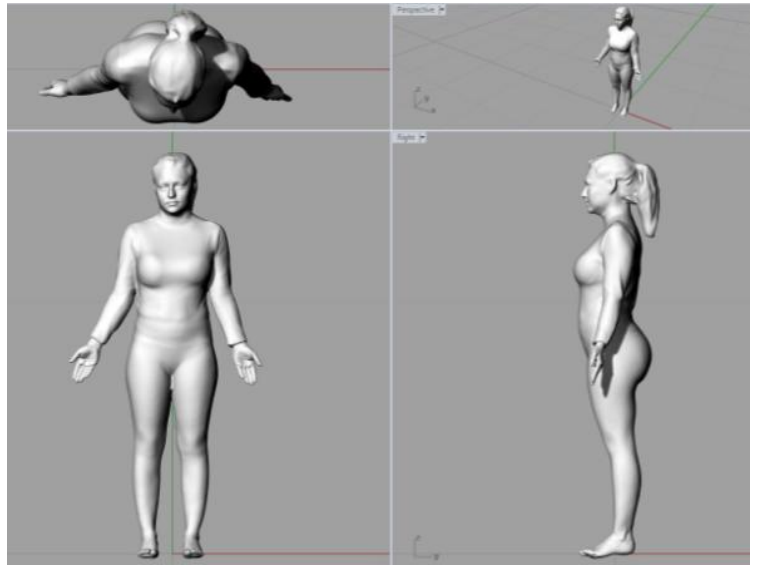

Fonte: Elaborado pelos autores.

O segundo participante, representando o percentil 95\% de altura masculino, prendeu o cabelo com uma touca para que este não interferisse nas medidas do rosto. A vestimenta utilizada pelo participante é uma sunga. A Figura 8 apresenta o modelo 3D das mãos dos pés, ambos referentes ao participante masculino. $O$ modelo 3D do corpo inteiro com os pés e as mãos inseridos no modelo 3D é apresentado na Figura 9.

Figura 8 - Modelo 3D da mão e do pé direito do participante masculino.

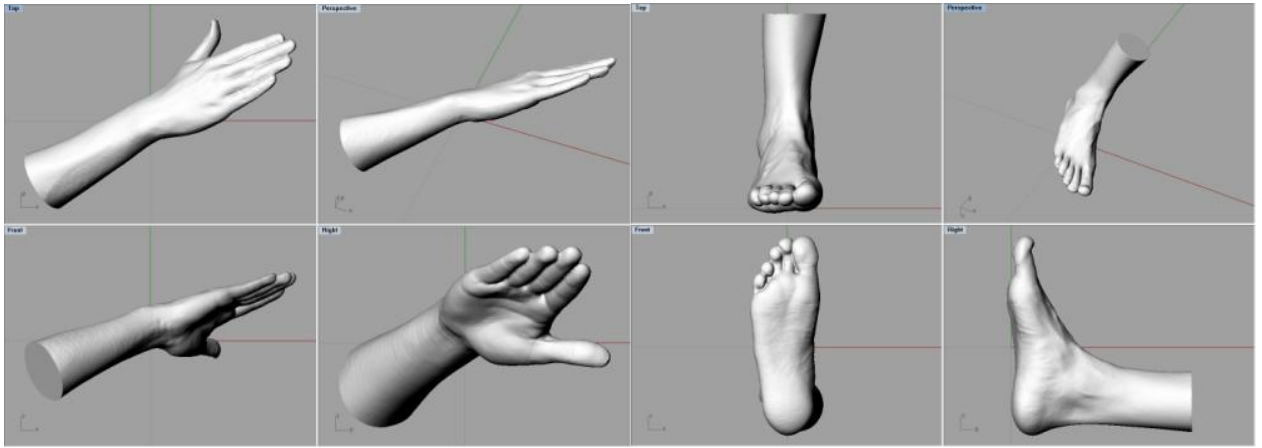

Fonte: Elaborado pelos autores.

Figura 9 - Modelo 3D do corpo inteiro do participante masculino.

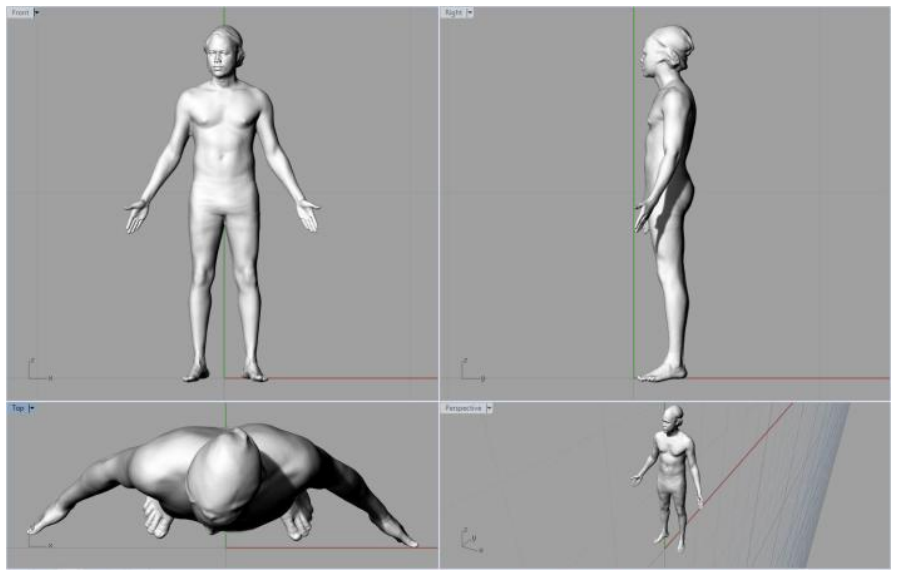

Fonte: Elaborado pelos autores. 
Para a obtenção dos valores das medidas antropométricas, os modelos 3D foram exportados em arquivo 'obj' e importados para o software de modelagem Rhinoceros ${ }^{\circledR}$ 3D. $\mathrm{O}$ processo de medição consiste em estabelecer as variáveis antropométricas em que serão mensuradas, assim como identificar estas variáveis no modelo 3D. As variáveis antropométricas estabelecidas para a presente pesquisa são baseadas entre os pontos anatômicos sobre as articulações do corpo, conforme as variáveis antropométricas sugeridas na Norma alemã DIN 33402, apresentadas por lida (2005). Sendo assim, são definidas as variáveis antropométricas entre as articulações dos membros inferiores como o comprimento e largura do pé, a articulação do calcanhar até a articulação do joelho, do joelho até a articulação do quadril, a estatura, a altura do ombro até o chão, largura dos ombros, largura dos quadris, articulações do ombro e cotovelo, cotovelo e punho e do punho até a extremidade do dedo médio.

Para realizar a medição, é necessário identificar as articulações e pontos anatômicos no modelo 3D. Para isso, são identificados os pontos de extremidade da malha tridimensional conforme os planos $x$, y e $z$ nas vistas ortográficas do software de modelagem. Portanto, após importar os modelos 3D, é necessário realizar o alinhamento no eixo $\mathrm{x}$, escalonar o modelo 3D para um tamanho adequado para a visualização e, se necessário, construir um eixo paralelo aos planos frontal, sagital e horizontal para a definição dos pontos de extremidade para aquisição dos valores das medidas. Os valores das medidas antropométricas da mão e do pé direito feminino são apresentados na Figura 10.

Figura 10 - Medição do modelo 3D feminino da mão e do pé direito.

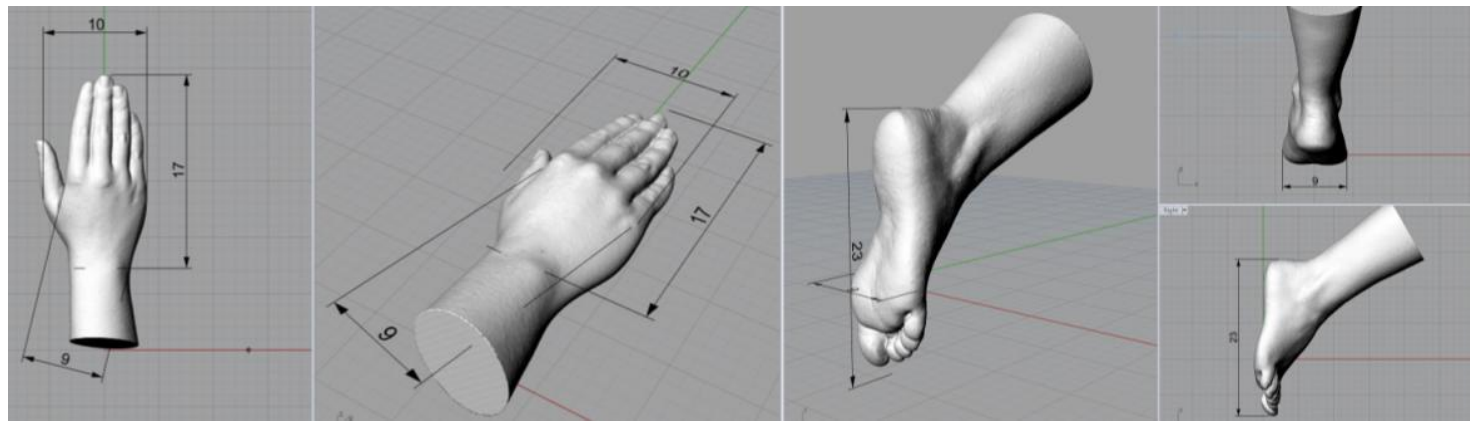

Fonte: Elaborado pelos autores.

Figura 11 - Processo de medição do modelo 3D feminino do corpo inteiro.

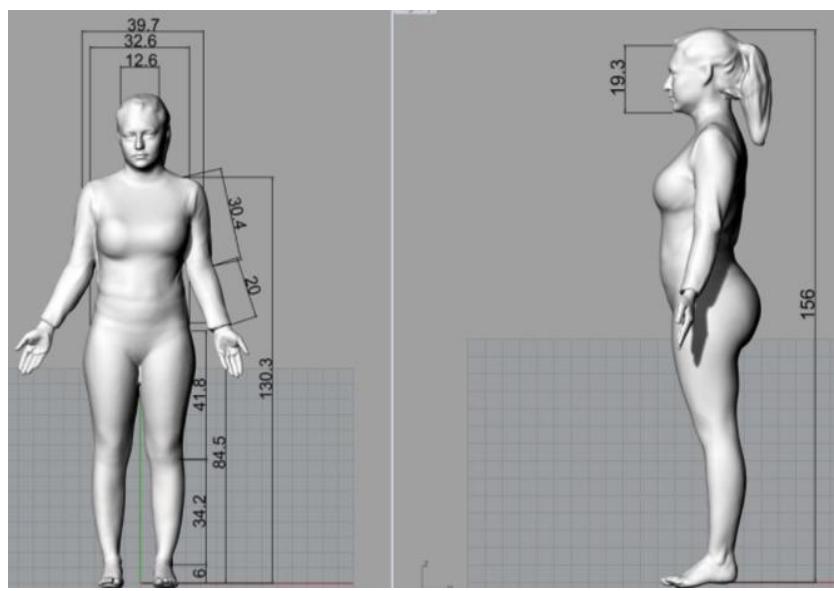

Fonte: Elaborado pelos autores. 
Os valores das medidas antropométricas do corpo inteiro são apresentadas na Figura 11 e, no Quadro 1, são descritas as variáveis mensuradas com os valores obtidos no modelo feminino.

Quadro 1 - Os valores das medidas antropométricas referentes ao modelo feminino.

\begin{tabular}{ll}
\hline VARIÁVEIS ANTROPOMÉTRICAS & $\mathrm{cm}$ \\
\hline Medidas do corpo em pé, ereto & 156,0 \\
\hline Estatura & 130,3 \\
\hline Altura dos ombros até o chão & 30,4 \\
\hline Articulação do ombro à articulação do cotovelo & 20,0 \\
\hline Articulação do cotovelo à articulação do punho & 39,7 \\
\hline Largura dos ombros & 32,6 \\
\hline Largura dos quadris & 41.8 \\
\hline Articulação do quadril à articulação do joelho & 84,5 \\
\hline Crista ilíaca até o chão & 34,2 \\
\hline Articulação do joelho à articulação do calcanhar & 6,0 \\
\hline Medida do calcanhar até o chão & 19,3 \\
\hline Variáveis da cabeça & 12,6 \\
\hline Medida do comprimento vertical da cabeça & 17,0 \\
\hline Medida da largura da cabeça, de frente & 9,0 \\
\hline Variáveis da mão & 23,0 \\
\hline Comprimento da mão (medida da articulação do punho até a ponta do dedo médio) & 9,0 \\
\hline Largura da mão (medida nas articulações da mão sem o polegar) & \\
\hline Variáveis do pé & \\
\hline Comprimento do pé & \\
\hline Largura do pé & \\
\hline
\end{tabular}

Fonte: Elaborado pelos autores.

O processo de medição da mão e do pé direito referente ao modelo 3D masculino é apresentado na Figura 12. O processo de medição do corpo inteiro referente ao modelo 3D masculino é apresentado na Figura 13. Os valores das medidas antropométricas referentes ao modelo masculino são descritos no Quadro 2.

Figura 12 - Processo de medição da mão direita no modelo 3D masculino.

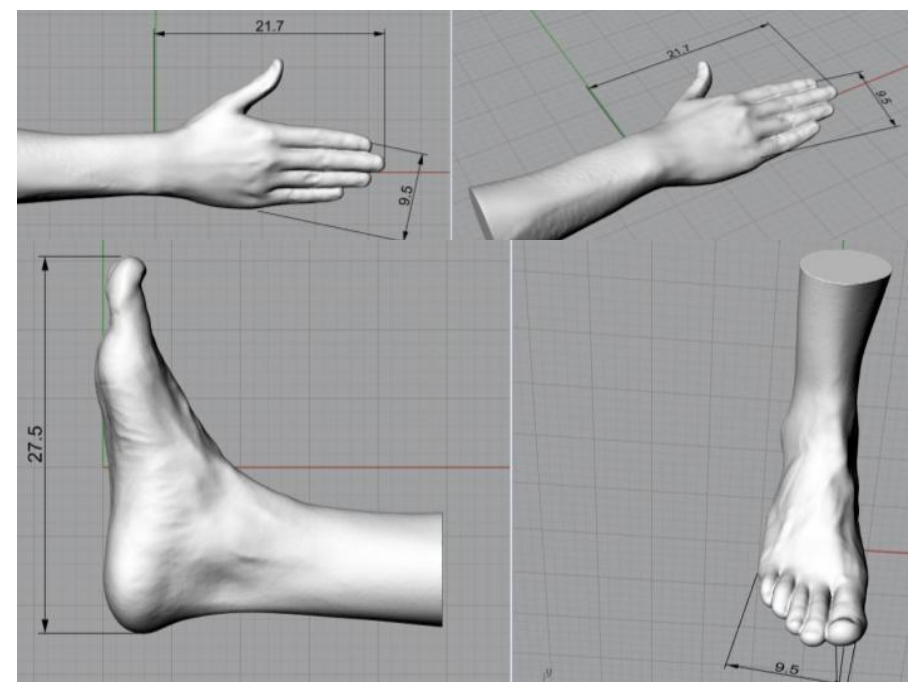

Fonte: Elaborado pelos autores. 
Figura 13 - Processo de medição do corpo inteiro no modelo 3D masculino.

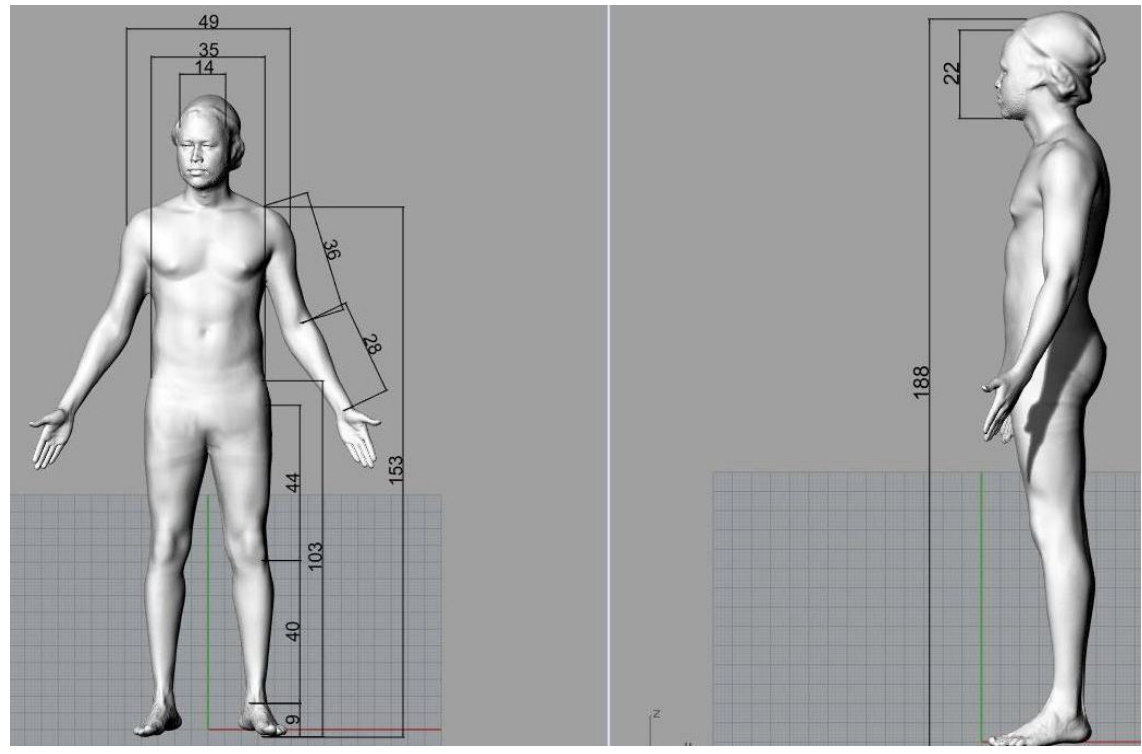

Fonte: Elaborado pelos autores.

Quadro 2 - Os valores das medidas antropométricas referentes ao modelo masculino.

\begin{tabular}{ll}
\hline VARIÁVEIS ANTROPOMÉTRICAS & $\mathrm{cm}$ \\
\hline Medidas do corpo em pé, ereto & 188,00 \\
\hline Estatura & 153,00 \\
\hline Altura dos ombros até o chão & 36 \\
\hline Articulação do ombro à articulação do cotovelo & 28 \\
\hline Articulação do cotovelo à articulação do punho & 49 \\
\hline Largura dos ombros & 35 \\
\hline Largura dos quadris & 103 \\
\hline Articulação do quadril à articulação do joelho & 44 \\
\hline Crista ilíaca até o chão & 40 \\
\hline Articulação do joelho à articulação do calcanhar & 9 \\
\hline Medida do calcanhar até o chão & 22 \\
\hline Variáveis da cabeça & 14 \\
\hline Medida do comprimento vertical da cabeça & 21,7 \\
\hline Medida da largura da cabeça, de frente & 9,5 \\
\hline Variáveis da mão & 27,5 \\
\hline Comprimento da mão (medida da articulação do punho até a ponta do dedo médio) & 9,5 \\
\hline Largura da mão (medida nas articulações da mão sem o polegar) & \\
\hline Variáveis do pé & \\
\hline Comprimento do pé & \\
\hline Largura do pé & \\
\hline
\end{tabular}

Fonte: Elaborado pelos autores.

\subsection{Inserção dos parâmetros no MHD e comparação do modelo 3D gerado pelo}

\section{Kinect.}

São inseridos os valores das variáveis antropométricas do participante feminino e do participante masculino, conforme as medidas dos Quadros 1 e 2 . As variáveis antropométricas 
paramétricas são delimitadas pelos pontos anatômicos e identificadas pelos landmarks. Para facilitar o processo de inserção dos valores das medidas antropométricas, o modelo 3D dos participantes é importado para o Blender e colocado lado a lado no MHD. Há dois modelos de MHD paramétrico, o feminino e o masculino, devido às diferenças na geometria do corpo entre eles, principalmente, no volume dos seios, do glúteo e da curvatura da lombar. O modelo 3D digitalizado da participante foi importado e colocado ao lado do MHD e os valores das medidas antropométricas foram inseridos (Fig. 14). A inserção dos valores do participante masculino foi realizada no MHD masculino paramétrico e o processo foi o mesmo utilizado anteriormente para o MHD feminino.

Figura 14 - Inserção dos valores das medidas antropométricas.
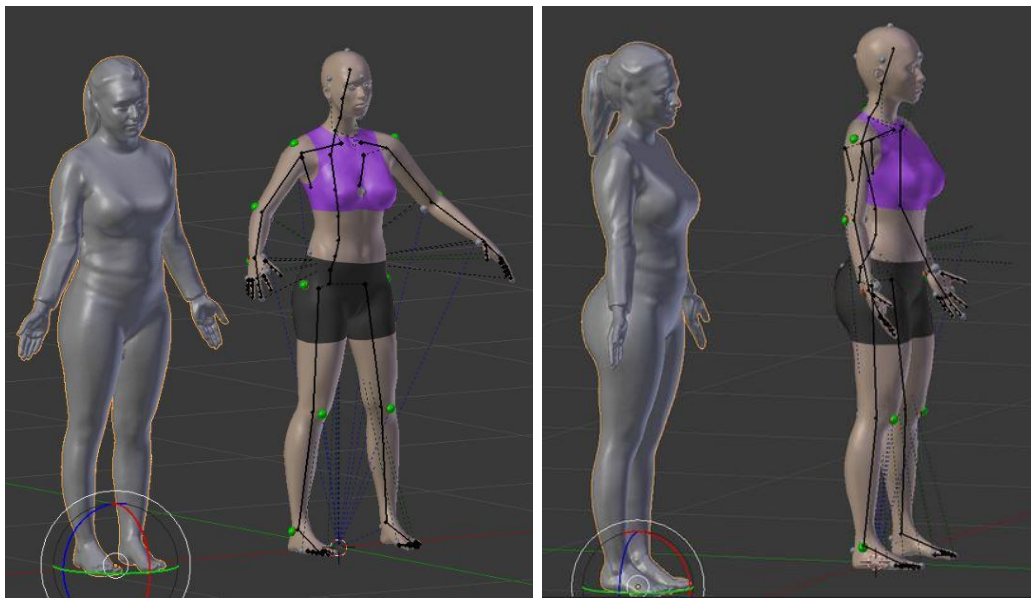

Fonte: Elaborado pelos autores.

A Figura 15 apresenta o modelo 3D digitalizado do participante masculino e, ao lado, o MHD sem a inserção das medidas das variáveis antropométricas. Ao lado, a imagem do MHD já com a aplicação das medidas antropométricas. São percebidas as alterações nos valores das variáveis antropométricas, conforme as medidas estáticas do participante masculino. Após a inserção das medidas antropométricas, o MHD Paramétrico feminino e o masculino pode ser utilizadas nas análises ergonômicas virtuais.

Figura 15- Modelo 3D do participante maculino e o MHD.
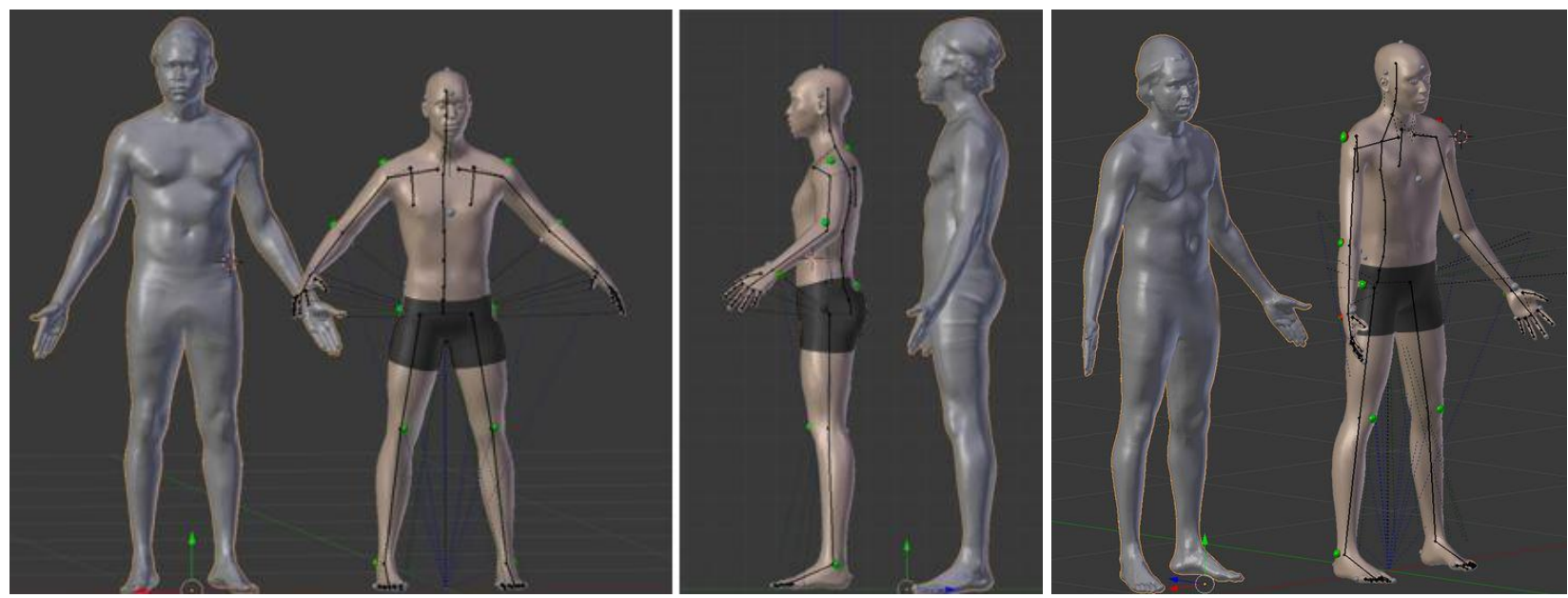
Fonte: Elaborado pelos autores.

\section{Resultados e Discussões}

O MHD paramétrico é um modelo humano virtual desenvolvido para ser utilizado nas análises ergonômicas em ambiente virtual. Este modelo possui particularidades fundamentais, pois possibilita a parametrização das variáveis antropométricas, obtenção das medidas estáticas e dinâmicas e avaliação do conforto postural durante a simulação virtual do uso do produto. Na Figura 16 é apresentado o MHD com as medidas antropométricas obtidas pelo método de Brendler (2013) realizando a análise ergonômica em uma estação de trabalho.

Figura 16- MHD com as medidas obtidas pelo modelo 3D feminino realizando análise ergonômica virtual.

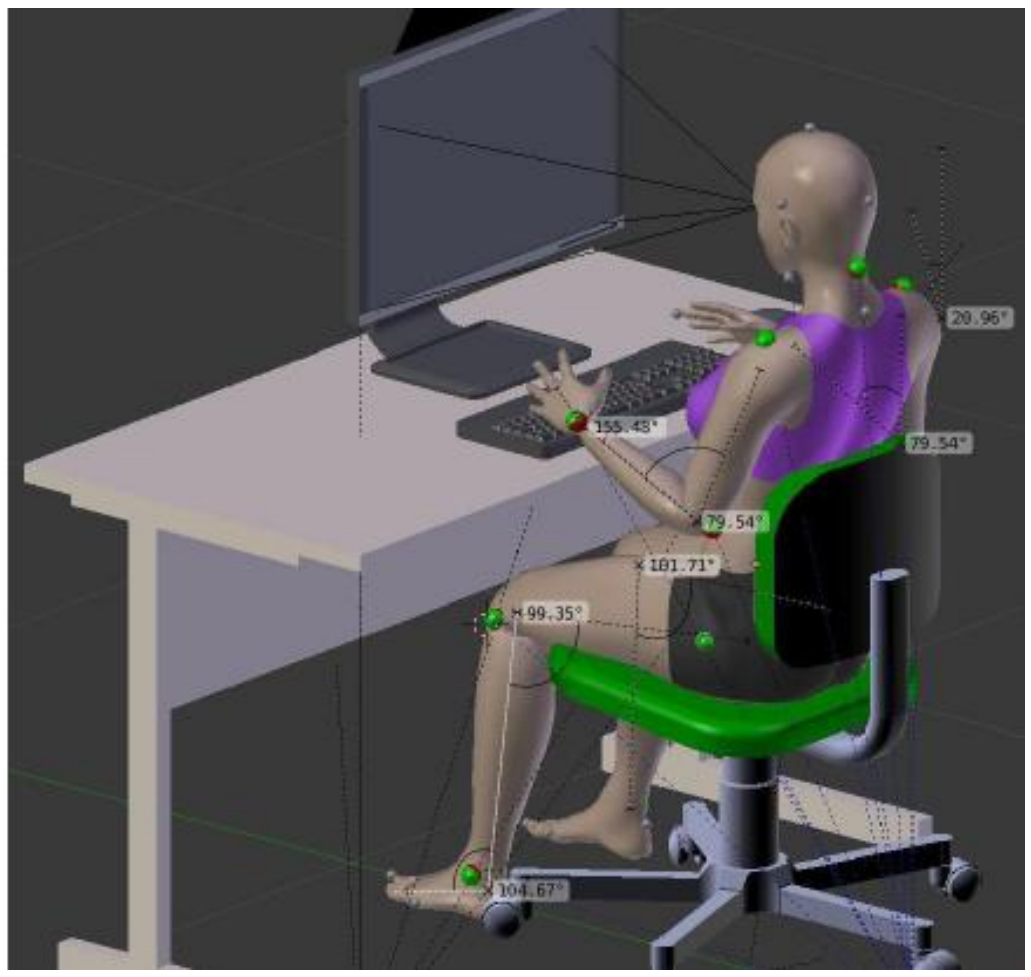

Fonte: Elaborado pelos autores.

A relevância da aplicação e da descrição do processo de obtenção de medidas estáticas pela digitalização 3D por um scanner de baixo custo está na necessidade de obter estas medidas de forma rápida, precisa e que auxilie o processo sem ter o contato físico com o usuário do produto.

Para facilitar o processo de análise ergonômica virtual com o uso do MHD, as medidas estáticas inseridas na primeira etapa do processo de desenvolvimento do produto, podem ser obtidas pelo processo utilizando a digitalização 3D ao invés de utilizar a medição manual. Muitas vezes a medição manual pode revelar medidas equivocadas pelo fato do corpo humano ter tecido mole e comprometer o processo de medição. Também, a falta de padronização dos pontos anatômicos utilizados como referência para o processo de medição (NORTON, 2005; SIMMONS, 2001). A geração de modelos humanos 3D já é realizada por diversos autores como Vezzetti e Marcolin (2012), Tomkinson e Shaw (2013) e Tong et al. (2012), porém estes utilizam 
digitalizadores 3D por um sistema a laser, o que aumenta excessivamente o custo para obtenção destes modelos (D'APUZZO, 2012).

\section{Considerações finais}

Algumas observações importantes relatadas no processo de medição pelo método de Brendler (2013) foi que o scanner 3D Kinect possibilita a geração do modelo 3D com alta definição do corpo inteiro em um tempo de apenas 2 minutos. Entretanto, para partes pequenas e com alta complexidade de geometria como as mãos e pés, o scanner perde a definição das malhas 3D. Para solucionar este problema foi utilizado o scannber de luz branca o que permitiu a geração do modelo 3D com alta definição da malha 3D. Para que se tenha uma melhor precisão das medidas, os participantes devem estar com uma vestimenta justa ao corpo, descalços, cabelo amarrado ou com uma touca.

Para obter as medidas antropométricas no modelo 3D, os autores seguiram o método descrito por Brendler (2013) sem dificuldades, assim como, a inserção destas medidas no MHD. O software Blender em que é utilizado o MHD desenvolvido para realização de análises ergonômicas possui um passo a passo explicando como efetuar a parametrização das variáveis do corpo bem como as medidas antropométricas. Os resultados podem ser observados nas Figuras 14 e 15, em que os modelos 3D gerados se tornam compatíveis com as medidas obtidas pelo processo de digitalização 3D. Assim, se pode afirmar que o método de Brendler (2013) é capaz de auxiliar no processo de análise ergonômica utilizando o MHD em ambiente virtual.

\section{Referências}

BLANCHONETTE, P. Jack Human Modelling Tool: A Review. Tech. Rep. DSTO-TR-2364, Defense Science and Technology Organization Victoria (Australia) Air Operations Division, Fishermans Bend, Victoria, Australia, 2010, document ADA 518132.

BRENDLER, Clariana Fischer. Modelo Humano Digital Paramétrico para análise ergonômica virtual no projeto de produto. 2017, p. 335. Tese de doutorado, Universidade Federal do Rio Grande do Sul, Escola de Engenharia, Programa de Pós-graduação em Design, Porto Alegre, RS.

BRENDLER, Clariana; TEIXEIRA, F. G. . Método para Obtenção de Medidas Antropométricas Utilizando um Digitalizador 3D de Baixo Custo. Revista Design \& Tecnologia, v. 11, p. 53-67, 2016.

BRENDLER, C. Método para levantamento de parâmetros antropométricos utilizando um digitalizador 3D de baixo custo. UFRGS, Porto Alegre, 2013, p.148. (Dissertação de Mestrado em Design- PgDesign da Universidade Federal do Rio Grande do Sul).

D'APUZZO, N. Recent Advances in 3D Full Body scanning with applications to fashion and apparel. In: Optical 3-d measurement techniques, 9., 2009,

HOBSON, D.; MOLENBROEK, J. Anthropometry and design for the disabled: Experiences with seating design for the cerebral palsy population. Applied Ergonomics, v. 21, n.1, p. 43-54, 1990.

IIDA, I. Ergonomia: projeto e produção. 2. ed. São Paulo: Edgard Blucher, 2005.

JONES, P.; RIOUX, M. Three-dimensional surface anthropometry: applications to the human body. Optics and Lasers in Engineering, v. 28, n. 2, p. 89-117, 1997. 
KOO B.-Y., PARK E.-J., CHOI D.-K., KIM J. J, CHOI M.-H. Example-based statistical framework for parametric modeling of human body shapes. Computers in Industry, v. 73, p. 23-38, 2015.

LU, J.; WANG, M. J. Automated anthropometric data collection using 3D whole body scanners. Expert Systems with Applications, v. 35, n. 1-2, p. 407-414, 2008.

LUXIMON, Y.; BALL, R.; JUSTICE, L. The 3D Chinese head and face modeling. Computer-Aided Design, v. 44, p. 40-47, 2012.

NORTON, K; OLDS, T. Antropométrica: um livro sobre medidas corporais para ovesporte e cursos da área da saúde. Porto Alegre: Artmed, 2005.

SANTOS, V.; ZAMBERLAN, M. C.; PAVARD, B.. Confiabilidade Humana e Projeto Ergonômico de Centros de Controle de Processos de Alto Risco. Rio de Janeiro: Synergia, 2009.

SIMMONS, Karla Peavy. Body measurement techniques: a comparison of threedimensional body scanning and physical anthropometric methods. Raleigh:vNCSU, 2001. (Tese de Doutorado. North Carolina State University).

TOMKINSON, G.; SHAW, L.; Quantification of the postural and technical errors in asymptomatic adults using direct 3D whole body scan measurements of standing posture. Gait \&Posture, v. 3, p. 172-177, 2013.

TONG, J; ZHOU, J; LIU, L; PAN, Z; YAN, H. Scanning 3d full human bodies using kinects. IEEE Transactions on Visualization and Computer Graphics, v. 18, n. 4, p. 643-650, 2012. Vienna. Anais

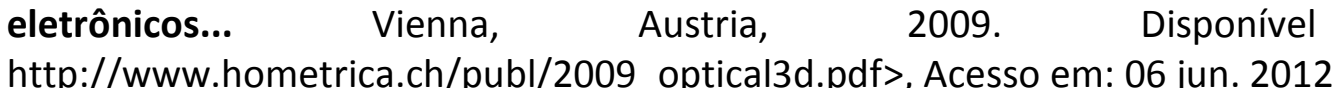

VEZZETTI, E; MARCOLIN, F. 3D human face description: landmarks measures andvgeometrical features. Image and Vision Computing, v. 30, n. 10, p. 698-712, 2012. 